\title{
The physical basis for recruitment variability in fish populations
}

\author{
B. J. Rothschild, T. R. Osborn, T. D. Dickey, and \\ D. M. Farmer
}

Rothschild, B. J., Osborn, T. R., Dickey, T.D., and Farmer, D. M. 1989. The physical basis for recruitment variability in fish populations. - J. Cons. int. Explor. Mer, 45: 136-145.

\begin{abstract}
Larval mortality is an important component of recruitment variability. To understand the mechanisms affecting larval mortality, it is necessary to take into account the nonlinear interactions involved in density-dependent population dynamics and the physical environment at scales of motion comparable with larval feeding scales. This paper develops a framework for further analysis by describing the population-dynamic and physical oceanographic setting for developing the notion of a "food signal", the fundamental unit of predator/prey interaction. This paper also considers the possibility that acoustic technology might be useful for making inferences about food signals.
\end{abstract}

B.J. Rothschild: University of Maryland, Center for Environmental and Estuarine Studies, Solomons, Maryland 20688, USA. T. R. Osborn: Chesapeake Bay Institute, Johns Hopkins University, Baltimore, Maryland 21218, USA. T. D. Dickey: Ocean Physics Group, University of Southern California, Los Angeles, California 90089 , USA. D. M. Farmer: Institute of Ocean Sciences, P.O. Box 6000, Sidney, B.C., Canada V8L $4 B 2$

\section{Introduction}

Many studies of fish recruitment concentrate on early life history, particularly larval nutrition and predation on larvae. A model of the interaction of predation and nutrition proposed by Shepherd and Cushing (1980) has not been tested or utilized, a task complicated by the fact that nutrition and predation mortalities are confounded.

But even if the contributions of larval starvation and predation could be easily resolved, other complexities need to be acknowledged. For example, important sources of recruitment variability may occur after the larval stage. In addition, all of these sources of variability depend upon the role of the physical environment. Understanding the role of the physical environment requires understanding a variety of physical-biological interactions over a very broad range of physical and biological scales.

The complexity of physical-biological interactions can only be made coherent through the development of a theory which takes into account the nonlinear nature of the "recruitment problem" and the relationship between population dynamics and the physical environment.

In working towards such a theory, it is necessary to minimize dependence on "artificial" nonlinearities which arise through the use of models that are based upon long causal chains. As an alternative, we believe that focusing on the shortest possible causal chain involving individual trophic transactions (see Rothschild, 1988) will do much to (a) clarify the way the system works, and (b) provide insights into the appropriate techniques to average or integrate individual ecosystem components.

This paper is concerned with a particular aspect of the problem, the physical basis for recruitment variability in fish stocks. We set the stage by considering the mechanisms of population stabilization at each phase of the life history. We then show that to understand these responses at the larval stage it is necessary to consider more than the simple ratio of predator density to prey density, which is the conventional approach; it is also necessary to understand the physical setting. Finally we consider how such a setting might be evaluated using acoustic techniques.

\section{Mechanisms of population stabilization}

Density-dependent responses to a population's food resources appear to be a primary population-stabilizing mechanism. When a population is at a high level of abundance it will "deplete" its food resources, reduce the amount of food per individual, reduce its growth rate, reduce its production of reproductive biomass, and 


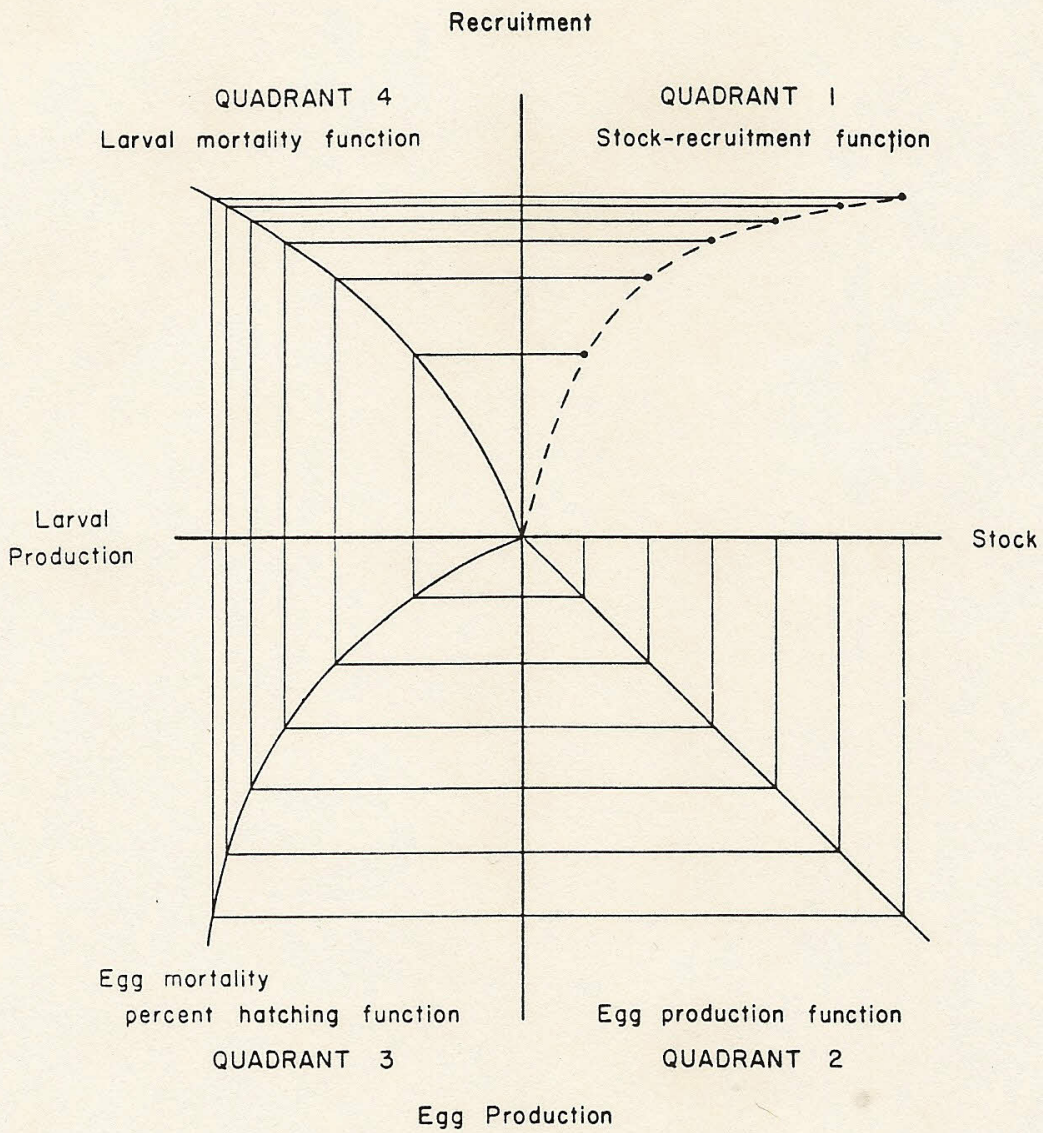

Figure 1. A Paulik diagram showing the construction of a recruitment/stock curve. Each quadrant contains a function representing a particular transformation in the recruitment/stock process. This example shows a linear density-independent stage in quadrant 2 and density-dependent stages in quadrants 3 and 4 . Note that the diagram is not drawn to scale. (From Rothschild, 1986; Figure 5.11.)

decline from its level of high abundance. When the population is at a low level of abundance the mechanisms operate in reverse and the population increases. Many other factors are, of course, involved and these may result in departures from this idealized picture; but nevertheless, the process generally seems to work, at least at extremely low and high food levels, as evidenced by the fact that in the short run it is rare to find populations that are either becoming extinct or exploding out of control to an enormous size.

The overall efficiency of stabilization is reinforced by the fact that each life-history stage comprises a set of stabilizing mechanisms generally independent of every other life-history stage. The redundant density-dependent mechanisms operating at each life-history stage are best illustrated by a Paulik diagram (Rothschild, 1986). The Paulik diagram (Fig. 1) decomposes the "transfer" between recruitment and spawning stock into functional relationships between spawning stock and egg production; between egg production and larval production; between larval production and juvenile production; and between juvenile production and recruitment. It draws attention to the importance of stages other than larvae. Two examples involving larval food and larval dispersal and the role of egg production serve to demonstrate the complexity of the relations.
Larval food and larval dispersal. It is well known that larval survival is enhanced by nutrition and diminished by predation. However, the full utility of food-requirement and predation-mortality estimates remains to be demonstrated, as what is important from a populationdynamics point of view are not the average values of these statistics but how they change with population density and the physical environment.

While density-dependent responses surely exist at all ontogenetic stages, it appears that some stages are more density-dependent than others. In order to appreciate the role of density dependence and the way that it affects various life-history stages it is important to appreciate the stabilizing or destabilizing role of each lifehistory stage. In this regard the traditional morphologically based separation of an egg and a larva into two life-history stages - an egg stage and a larval stage - may be misleading from a functional population-dynamics classification. This is because from a population-dynamics point of view, the egg and "early" larvae appear to behave differently from the late-larvae or post-larvae, suggesting that it would be appropriate to think of the overall egg/larval phase as having two parts, namely (a) an egg and very-early larval stage, and (b) a larval stage proper thereafter, and until metamorphosis to the true juvenile stage of development. The transition be- 
tween the two larval phases probably coincides with the opening of the alimentary canal and first-feeding; in European terminology these two larval stages are indeed distinguished by the terms "larval" and "post-larval".

In this regard, it seems as though during the initial phases of the egg/larval stage the eggs-and-larvae disperse to "sample" the environment or to otherwise "find" salubrious living conditions and to minimize density-dependent interactions. This is the way fish have evolved to take advantage of the patchy nature of food, a component of the extreme variability in environmental conditions, at the scales of larval existence. During ontogeny, each larval fish occupies a volume that may or may not contain conditions where there are adequate nutrition and minimal predation. If conditions are salubrious then the larval fish survives; otherwise, the larval fish dies. Conditions which are not salubrious can arise from a variety of quite different causes. For example, risk to larvae is increased by inadequacy of food and intense predation. However, ordinarily adequate food and little predation become disadvantageous with unusually high densities of larvae, or when there are physical factors affecting larvae negatively but their predators positively.

Thus the early life history might be divided into two stages. In the earliest, dispersal is the critical biological phenomenon; in the second, density dependence is the critical phenomenon. In order to study the interaction between the larvae and the physical setting it is necessary to separate the roles of dispersal and density dependence.

Egg production. Evidence continues to mount that egg production plays an important role in contributing to population variability (Rothschild, 1986, chapter 6; Almatar and Bailey, 1989, this issue, pp. 113-124). Rothschild and Fogarty (1989, this issue, pp. 131-135) point out that the variability in the magnitude of spawningstock biomass is greater than the magnitude of the variability in egg production. They also note that the existence of this phenomenon had important implications for the many management-related stock-assessment techniques that use spawning-stock biomass (SSB) as a proxy for egg production, because if the correct values of egg production were actually used, then different management conclusions would be drawn.

Rothschild and Fogarty's proposition that egg production over a large range of population sizes is relatively stable has two important implications for the study of population variability.

1) While egg production is usually the most important locus of population stability, the larval stage is usually the most important locus of population variability.

2) Deterioration of the egg-production density-dependent stabilizing mechanisms can provide an important contribution to population collapse (see, e.g., Rothschild, 1986, pp. 224-225).

\section{Alternatives to the density-measurement approach}

One of the difficulties in trying to understand the dynamics of the egg-and-larval stage is that the study of the interrelationships among organisms has conventionally been constrained to consider only the relative densities of predator and prey organisms and not their relative contact and ingestion rates, which are not necessarily proportional to their relative densities (see, e.g., Holling, 1965, or Rothschild, 1988). In other words, the population-dynamics response depends upon factors other than the simple ratio of predator density to prey density. This can easily be seen because the populationdynamics response depends on the number of prey ingested per predator and this ratio is in general different from the ratio of prey contacted per predator. And in turn, the ratio of prey contacted per predator can be quite different from their relative densities (Rothschild and Osborn, 1988).

The problem is that the inferred contact rate and the consumption rate depend upon not only the density of prey and predator as measured, for example, by plankton nets, but also the relative scales of plankton-net sampling and larval feeding; the velocity of the predator relative to prey; the variance of prey distribution relative to the predator; and the so-called functional response of predators to various densities of prey. To complicate matters further, each of these factors is heavily dependent upon the physical structure of the environment.

\section{Scales}

The distribution of biological properties seems everywhere "patchy". This means that estimates of plankton density (numbers per unit volume) are a complicated function of the distribution of sampled volumes and the configuration of patches. But clearly the pattern of the three-dimensional distribution of patches is linked with the kinetics of the water in which the plankton are embedded.

This suggests that if all other things are equal, the physical structure can alone dominate patchiness and hence the interaction of organisms. It further suggests that sampling schemes which are optimal in some sense for one turbulence regime, are unlikely to be optimal for another turbulence regime.

Finally, even though an optimal sampling structure can be linked with a turbulence regime, there is probably a great disparity between inferences on density made from conventional sampling devices and the ingestion and contact rates of organisms simply because 
sampling is usually conducted on scales unrelated to the scales of feeding. In other words, traditional sampling processes tend to average out the variability that must be taken into account in the analysis of feeding.

\section{Relative velocity of predator and prey}

Contact rates between predator and prey are only partially dependent on the relative density of predator and prey; they depend as well upon the relative velocity of predator and prey which is a function of small-scale turbulence (Rothschild and Osborn, 1986, 1988). (This point is emphasized in Yamazaki and Osborn, 1988; for application see Rothschild, 1988.)

\section{Second-order distribution of prey}

The theory of point processes (see, for example, Cox and Isham, 1980) takes into account both the mean and the variance of a distribution of events. The mean is called a first-order property while the variance is called a second-order property. The second-order property of prey contact can have effects that substantially influence the nature of contact and ingestion, given contact.

First, with regard to the nature of contact, a patchy prey distribution will alter the nature of contact between predator and prey by increasing the variance of the contact rate. If the variance is low, then, all other things being equal, the predator will destroy its prey in a "uniform" manner. Now if the mean number of prey is kept the same and the variance of the contact rate increased, some predators will encounter high concentrations of food and others low concentrations of food. Circumstances could arise where the reproductive processes for certain predators would be diminished given the time constants for elaboration of reproductive biomass; at the same time certain prey would find refuge in high-variance situations.

Second, with respect to ingestion, given contact, there is the possibility of the familiar predator/prey functional response where the predator reduces its ingestion per unit prey at high prey densities. If prey density were uniformly high, then the functional response might follow the typical response described in the literature where the rate of ingestion declines as the abundance of prey increases. On the other hand, if prey were patchy, then the functional response would depend on the mean distance between patches and the numbers of organisms in each patch. This suggests that even though the mean number of prey is constant, the nature of the functional response, and hence the ingestion rate, would vary as a function of patchiness.

Again, while one component of patch aggregation and dissipation is due to the behavior of the organisms, another component depends on the turbulent motions on feeding scales; thus, high levels of turbulent motion could create patches at feeding scales independent of the spatial distribution of predator and prey.

\section{Food signals}

Rothschild (1988) has pointed out that the individual trophic transaction can be considered in terms of "food signals". In this section the notion of "food signal" is made more precise. A particular food signal refers to (a) the temporally defined encounter between a particular predator and its prey, and (b) the ingestion of prey by the predator given a particular frequency of encounter.

A particular food signal or realization of a food signal is constructed by defining (1) a prey-encounter realization, and (2) an ingestion realization, which clearly must be a function of a corresponding encounter realization.

We write the (i)th contact realization as

$$
C_{i}=\left(c_{i 1}, c_{i 2}, \ldots, c_{i N}\right) \quad \begin{aligned}
& i=1, \ldots, P \\
& j=1, \ldots, N
\end{aligned}
$$

where $\mathbf{P}$ is the number of realizations in an "experiment" and $\mathrm{N}$ is the number of discrete time intervals chosen for study. Let each $\mathrm{c}_{\mathrm{ij}}$ equal one or zero depending upon whether or not an ingestible prey is contacted.

We define the (i)th ingestion realization corresponding to the (i)th contact realization as

$\mathrm{S}_{\mathrm{i}}=\left(\mathrm{s}_{\mathrm{i} 1}, \mathrm{~s}_{\mathrm{i} 2}, \ldots, \mathrm{s}_{\mathrm{iN}}\right)$

where $i$ and $j$ are the same i's and j's as in (1). The $s_{i j}$ 's take on the values one or zero depending upon whether or not a prey is ingested. Since $\mathrm{S}_{\mathrm{i}}$ depends directly on its

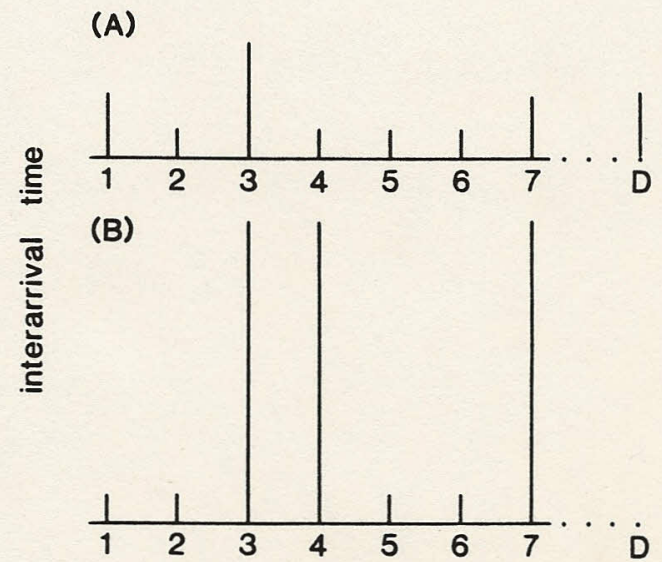

Figure 2. Interarrival times (the time between events) for the $1 \ldots$ (D)th prey as generated by two different mixes, $M$, of variables. Note that $(A)$ is a random distribution where $(B)$ is clumped. 


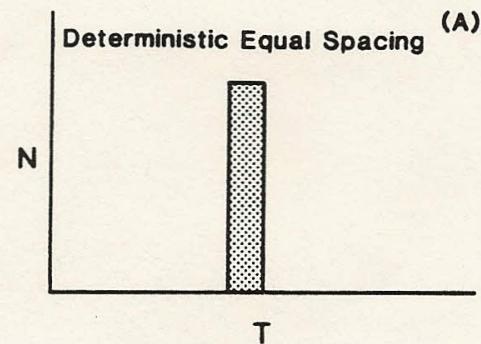

$T$

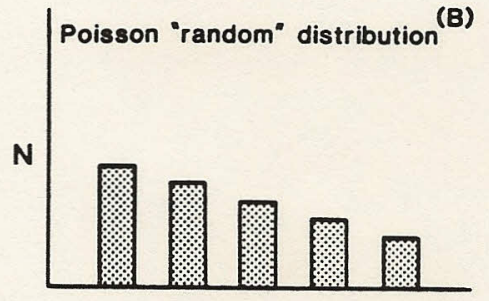

$T$

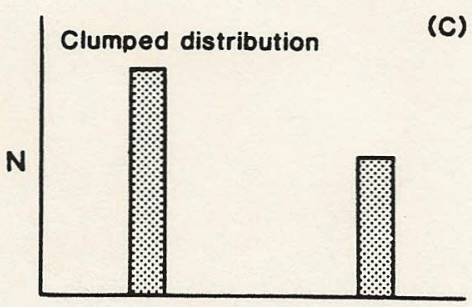

$T$

Figure 3. Alternative forms of waiting-time distributions. (A) is deterministic; (B) and (C) correspond to Figure 2.

corresponding $\mathrm{C}_{\mathrm{i}}, \mathrm{s}_{\mathrm{ij}}$ can only equal one if its corresponding $\mathrm{c}_{\mathrm{ij}}$ equals one.

Definitions (1) and (2) suggest other definitions. For example, the density of prey for the (i)th realization is

$D_{i}=\sum_{j} c_{i j}$

Now note that even with $\mathrm{D}_{\mathrm{i}}$ constant the distribution of the waiting times between adjacent prey is a function of, inter alia,

turbulence regime, $T_{k}$,

the relative velocity of predator and prey

independent of water motion, $\mathbf{R}_{k}$,

the spatial configuration and density of prey, $D_{k}$,

and

ingestion rules, $I_{k}$,

where the subscript $\mathrm{k}$ refers to the (k)th experiment.

We can, depending upon the values of $M=$ ( $T, R, D, I)$, envisage different distributions of waiting times as functions of contact rate. This is shown in Figure 2 for an upper and a lower bound case, i.e., a random and a clumped distribution.

These arrival times generate a frequency distribution which is plotted in Figure 3. These distributions pose the question of the form of the ingestion realization or signal. As is well known, not all predators eat every prey they encounter because of saturation or functionalresponse effects. Examples of the sorts of effects that might affect the numbers of prey given ingestion are shown in Figure 4, which shows how mortality and survival of predator and prey are "traded-off" as a function of environmental conditions.

These considerations make it possible to design a simulation experiment. For example, consider two mixes of environmental conditions, $\mathbf{M}_{1}$ and $\mathbf{M}_{2}$. For simplicity, let $M_{1}$ and $M_{2}$ be the same except that $M_{1}$ is a high-turbulence regime and $\mathbf{M}_{2}$ is a low-turbulence regime. Suppose 1000 realizations are conducted under conditions $M_{1}$ and 1000 realizations under $M_{2}$. This would result in $1000 \mathrm{~S}_{\mathrm{i}}$ 's under $\mathrm{M}_{1}$ and $1000 \mathrm{~S}_{\mathrm{i}}$ 's under $M_{2}$. The frequency distribution of the numbers of realizations in which the predator ingests $1,2 \ldots$ prey under $M_{1}$ and $M_{2}$ could then be plotted and might look like Figure 5.

\section{(A)}

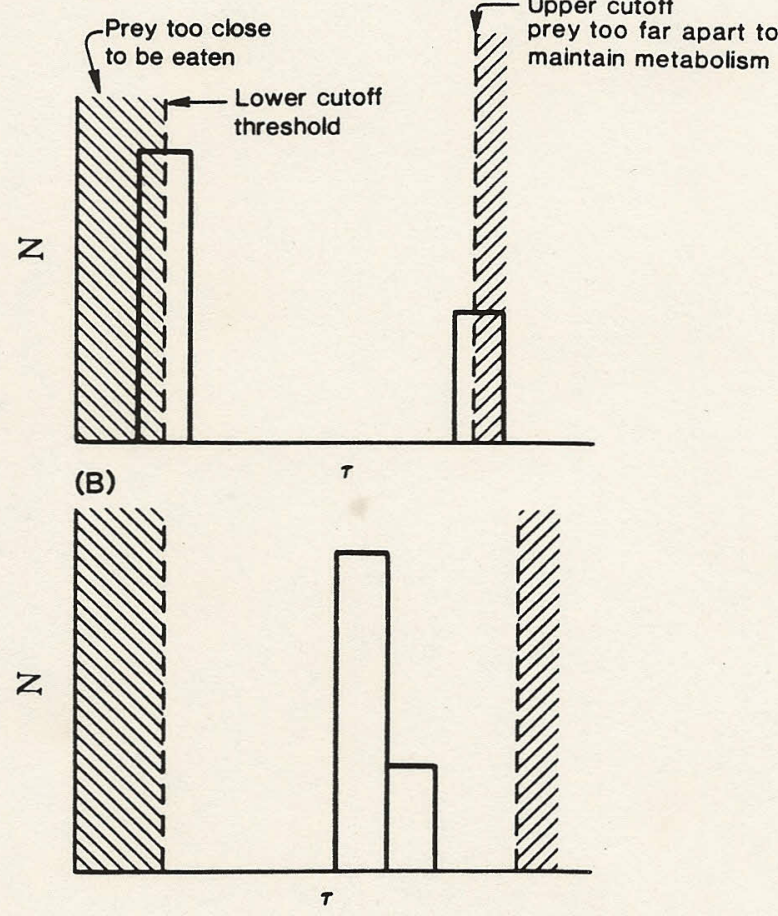

Figure 4. In panel (A) prey are too close together to be eaten efficiently or too far apart to maintain metabolism. In panel (B), $M$ changes so even though D is the same in (A) and (B), environmental conditions increase prey survival to the liability of the predator in (A) and vice versa in (B). Note that the form of the lower threshold is a more complicated nonlinear function of prey density.

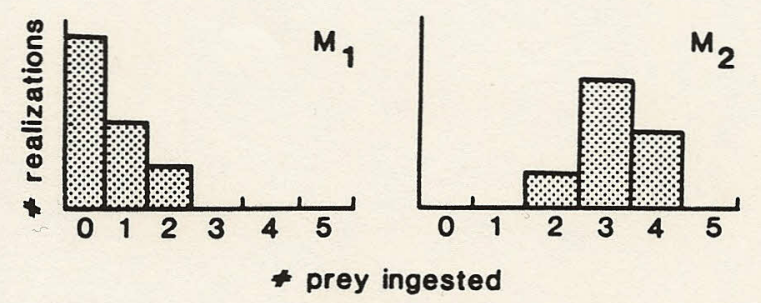

Figure 5. Frequency distribution of number of prey ingested as a function of environmental conditions (e.g., turbulence). 


\section{Physical structure}

Two sorts of problems are of particular concern. The first involves turbulence at feeding scales, as it is likely that these motions have an important effect on the contact rates of predator and prey. The second involves localized low-Reynolds number effects. These effects play an important role in the ingestion-given-contact problem.

\section{Turbulence}

A turbulent flow field has the following general qualitative attributes: (1) it is diffusive and dissipative, (2) it is irregular and its velocity fluctuates, (3) it is intermittent and patchy, and finally (4) its Reynolds number is high. The Reynolds number is the ratio of the inertia to the viscous term in the Navier-Stokes equation and is a useful figure of merit for roughly distinguishing turbulent from laminar flow conditions. Consider a turbulence energy spectrum (Fig. 6, after Hinze, 1959), which indicates certain important scales and features. The larger-scale turbulent eddies (smaller wavenumbers) depend on the conditions of the generation of the turbulence; however, as the energy cascades to the smallerscale eddies, the energy spectrum becomes universal. The peak in the spectrum indicates the scale of the most energetic eddies, and with greater wavenumber, the inertial and finally viscous subranges are encountered. The transition from turbulent to laminar motion is marked by the Kolmogorov length scale, or the Kolmogorov wavenumber $\mathbf{k}_{\mathrm{d}}$.

There is a considerable amount of information now available concerning certain aspects of turbulence in the upper ocean. One of the important parameters obtained from ocean measurements is the dissipation rate of tur- bulent kinetic energy, $\varepsilon$. A summary of values obtained from various oceanic conditions has been presented by Lueck and Reid (1984). A few representative ranges of values of $\varepsilon$ are presented in Table 1. From these data, the corresponding Kolmogorov length scale, $\eta$, and the Kolmogorov velocity scale, $v_{k}$, ranges have been calculated and are also shown in Table 1 . In addition, Reynolds numbers have been computed for a passive copepod zooplankton and a diatom using the Kolmogorov velocity scale and the approximate sizes of the organisms along with the kinematic viscosity, $v$.

It can be seen that the characteristic Reynolds numbers as based upon the small-scale (Kolmogorov scale) flow are quite small $(0(1)$ or less) and that the Reynolds numbers of the zooplankton are two orders of magnitude greater than those of the diatoms. If one considers the swimming speed of a zooplankton copepod to range between 0.1 and $10 \mathrm{~cm} \mathrm{~s}^{-1}$, Reynolds numbers ranging from 1 to 100 can be obtained. One of the important points here is that Reynolds numbers relevant to this problem vary by as much as five orders of magnitude. Furthermore, the drag coefficient varies by a factor of 20 over this range of Reynolds numbers. On this basis, the motion of the predator and prey would be expected to differ, and thus a relative motion would exist.

Davies (1972) has noted that large particles will follow the large-scale motions only; however, if the particles are of the same size as the smallest scales of motion, they will follow these scales. It can be seen from Table 1 that the diatoms are generally two orders of magnitude smaller than the Kolmogorov wavelength, while the zooplankton are within an order of magnitude of the Kolmogorov wavelength, implying that they may or may not satisfy Davies' general requirement. Thus, it may be concluded that the predator/prey interaction process takes place in a transitional flow regime.

There are also fundamental questions concerning the

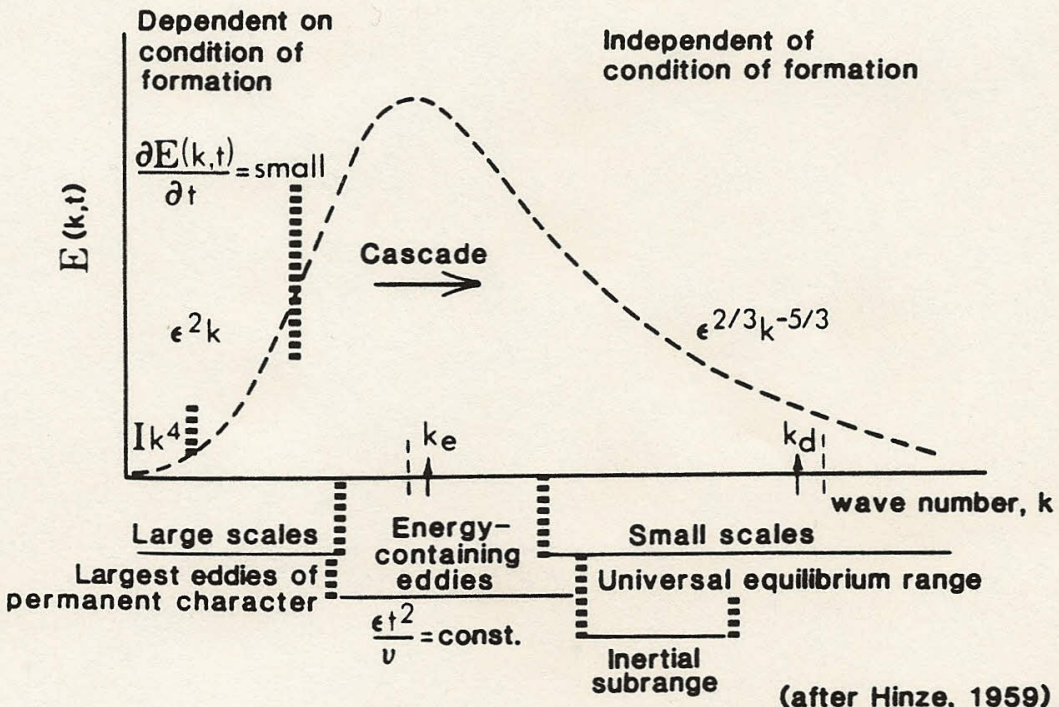

Figure 6. A typical three-dimensional turbulence energy spectrum function where $\mathrm{k}=$ wavenumber, $\varepsilon=$ energy dissipation rate, $k_{e}=$ wavenumber of energy containing eddies, $k_{d}=$ Kolmogorov wavenumber, $\eta=$ Kolmogorov wavelength scale, and $v_{\mathrm{x}}$ $=$ Kolmogorov velocity scale. 
Table 1. Turbulence scales as related to predator/prey problems.

\begin{tabular}{|c|c|c|c|c|c|}
\hline Region & $\begin{array}{c}\varepsilon \\
\left(\mathrm{cm}^{2} \mathrm{~s}^{-3}\right)\end{array}$ & $\begin{array}{c}\eta \\
(\mathrm{mm})\end{array}$ & $\begin{array}{c}v_{k} \\
\left(\mathrm{~mm} \mathrm{~s}^{-1}\right)\end{array}$ & $\mathrm{Re}_{\mathrm{z}}$ & $\mathrm{Re}_{\mathrm{d}}$ \\
\hline $\begin{array}{l}\text { North Pacificic }{ }^{\mathrm{a}} \ldots \ldots \ldots \ldots \ldots \ldots \ldots \ldots \ldots \ldots \ldots \\
\text { Scotia Shelf }{ }^{\mathrm{b}} \ldots \ldots \ldots \ldots \ldots \ldots \ldots \ldots \\
\text { Seym. Narr. }{ }^{\mathrm{c}} \ldots \ldots \ldots \ldots \ldots \ldots\end{array}$ & $\begin{array}{l}10^{-6}-10^{-2} \\
10^{-3}-10^{-2} \\
10^{-3}-1\end{array}$ & $\begin{array}{c}10-1 \\
2-1 \\
2-0.3\end{array}$ & $\begin{array}{l}0.1-1 \\
0.6-1 \\
0.6-3\end{array}$ & $\begin{array}{l}0.1-1 \\
0.6-1 \\
0.6-3\end{array}$ & $\begin{array}{l}0.001-0.01 \\
0.006-0.01 \\
0.006-0.3\end{array}$ \\
\hline
\end{tabular}

${ }^{a}$ Lueck and Osborn (1982).

${ }^{\mathrm{b}}$ Oakey and Elliot (1980).

${ }^{\circ} \mathrm{G}$ rant et al. (1962).

Zooplankton Reynolds number: $\operatorname{Re}_{2}=\frac{v_{\mathrm{k}} \mathrm{d}_{z}}{v}$

where $d_{2} \sim 1 \mathrm{~mm}$ for common copepod zooplankton.

Diatom Reynolds number: $\operatorname{Re}_{d}=\frac{v_{k} d_{d}}{v}$

where $d_{d} \sim 0.01 \mathrm{~mm}$ for diatom.

Swimming copepod Reynolds number: $\operatorname{Re}_{\mathrm{s}}=\frac{\mathrm{Ud}_{\mathrm{z}}}{\mathrm{v}}$.

For swim speeds of 0.1 to $10 \mathrm{~cm} \mathrm{~s}^{-1}, \operatorname{Re}_{\mathrm{s}} \sim 1$ to 100 .

motion of a particle relative to a steady, uniform fluid flow. This problem has been considered extensively for the idealized case of a sphere with varying diameter or Reynolds number. The very low Reynolds number case $(\mathrm{Re} \ll 1)$ gives the Stokes' Law form; however, for greater Re appreciable departure from Stokes' Law occurs and more complicated Re-dependent empirical forms must be used. Furthermore, little work has been done on non-spherical shapes and surface roughness effects.

Because of the importance of the general problem, there has been a variety of particle-dynamics research with applications to chemical reactions and combustion, sediment transport, and atmospheric aerosols. Another stimulus for research on this topic is the use of particles for tracers of fluid flow (e.g., Dickey and Mellor, 1982). There have been a few laboratory studies covering rather limited dynamical ranges devoted to sedimentsettling velocities in oscillating and turbulent flows. In addition, there have been wind-tunnel experiments using particles of varying density and sizes.

Nonetheless, our ability to model particle motion in oscillating and turbulent flows is still quite limited. One of the more relevant studies was done by Granata and Horne (1985), who examined the horizontal dispersion rates of two diatoms (one large and one relatively small). They found the two rates to vary significantly and hypothesized that this variance was caused by the difference in the sinking rates. In some ways, our particular problem is simpler than those pursued by others (e.g., combustion research involves many reactants), and in some ways, it is more complex (e.g., the feeding behavior aspect).

\section{Localized physical effects}

The localized physical effects involved in the problem of ingestion apply at the smallest scales in diffusive transport very close to a plankter. The localized effects involve a range of topics from diffusive mixing to chemoreception. The ambient flow field of relevance to the individual predator/prey interaction is in the small-scale ranges described earlier. In a study related to the interpretation of particle-tracer turbulence data, Dickey and Mellor (1979) rederived the Kolmogorov $r^{2 / 3}$ law using high wavenumber spectra. In this work, they presented a formulation for Re number-dependent velocity correlation functions which appears to be applicable to the present circumstance of small-scale flow.

Finally, the role of feeding currents in the predator/ prey problem has been elucidated recently. Strickler (1985) has written an excellent review on this subject and suggested that small-scale perturbations of feeding currents by ambient fluid motions can affect the capture of food by animals such as calanoid copepods.

\section{The potential for acoustic measurements}

The foregoing discussion raises a number of formidable observational challenges. How can contact rates be determined? What are the behavioral responses to turbulence? How do zooplankton respond to the presence of a predator? The sensitivity of zooplankton to the presence of instrumentation dictates a remote-sensing approach. While optical detection undoubtedly has possibilities, there are practical difficulties in using optical 
systems except at very close range. Moreover, zooplankton are sensitive to light. Acoustic techniques appear to offer the best promise for tackling some of these problems.

While acoustic backscatter from zooplankton has been studied for many years, the range of measurement concepts that have been applied remains limited. The use of acoustic techniques to study behavior patterns and interactions with variable physical processes has received relatively little attention. We identify five different approaches to acoustic measurement and suggest ways in which they might contribute to the observation and quantitative measurement of zooplankton distribution and behavior.

\section{Target strength}

This is the approach that has received most attention in the past (see, for example, Andersen and Zahuranec, 1977). An interesting development along these lines, following early work of Cushing (1980), is the use of several different acoustic frequencies (Holliday and Pieper, 1980, 1984). The target strength at different frequencies provides additional degrees of freedom in characterizing the acoustic properties of the targets. For example. if two sizes of zooplankton are known to be present, each having a distinct and known frequency dependence of target strength, then the relative abundance of each can, in principle, be determined from the observed backscatter data.

A difficulty with this approach, common to all backscatter acoustic measurement techniques, is that the interpretation depends on an adequate model for the acoustic properties of the targets. For example, in the case just described, it has been assumed that there are just two types of targets, and that their acoustic properties are known. A continuous size distribution, or inadequate information about the acoustic scattering properties, precludes a unique interpretation of the signal. Another complication is the anisotropy of plankton target strength with respect to orientation. Nevertheless, target-strength measurements are at present the most basic and useful of acoustic techniques for studying plankton.

\section{Statistical distributions of target strength}

Apart from the mean target strength of a plankton distribution, there is also information contained in the statistical distribution of the signal. The use of backscatter-strength fluctuations to count the number of targets in a given volume has been discussed by Wilhelmij and Denbigh (1973) and Denbigh and Weintraub (1986). The motivation for this approach was the development of fish stock assessment techniques that were independent of a knowledge of fish target strength. The tech- nique, however, is equally applicable to zooplankton measurement; simple relationships exist between the second moment of the intensity and the number density. Clay and Heist (1984) and Stanton and Clay (1986) have considered other aspects of the statistical distribution that can provide useful information about the target distributions; again, the motivation here was the study of fish (and sea-floor irregularities), but the ideas have equal applicability to zooplankton measurement.

Of particular interest are the signals composed of overlapping echoes. The small size and often dense distribution of zooplankton make it difficult to interpret high-frequency backscatter signals. Once the number density becomes relatively high, the individual targets start to merge and the accurate estimation of statistical properties becomes difficult.

\section{Statistical distributions of backscatter phase}

Farmer and Huston (1988) presented a new approach to the acoustic measurement of number density, utilizing the phase statistics. In contrast to the statistical methods discussed above, data from successive transmissions are coherently processed. It can be shown that the second moment of the phase is related to the number density of targets in the sampled volume. But the coherent summation of successive echoes leads to a decreasing second moment, as the summed number density increases. This leads to a sensitive measurement of number density.

The determination of zooplankton distributions that might be based on the phase statistics depends on having an appropriate scattering model. If the plankton are of size comparable to, or greater than, the acoustic wavelength, they cannot be treated as point scatterers. This suggests the use of multiple frequencies to provide additional discrimination. More generally, the complex echo signal, coherently processed, should lead to estimates of the complex scattering properties of the targets. While this approach is as yet in its infancy and remains to be tested, it offers promise for the development of sensitive new measurement techniques for studying zooplankton.

\section{Coherent Doppler}

Coherent Doppler measurement, in which coherent processing of backscatter from several pulses is used to estimate rate of phase change, has been developed as a tool for remote measurement of current profiles. While the small-scale distribution of currents, especially current shear, is of great interest in zooplankton studies, an interesting possibility of determining the statistics of movement of individual plankton relative to their neighbors arises from the calculation of the decorrelation time of the (complex) echoes (Farmer and Huston, 
1988). The decorrelation of the signal, or equivalently, the bandwidth or second moment of the Doppler spectrum, is related to the second moment of the random component of motion resolved along the acoustic axis.

As with the other backscatter measurements, satisfactory interpretation of this signal requires a suitable acoustic scattering model. But the determination of random components of motion in this way appears to be a viable approach to the difficult problem of estimating contact rates of zooplankton in a given distribution of phytoplankton. If the random motion, due to swimming motions directly associated with feeding, can be distinguished from the mean motion which results from advection, then in the absence of turbulence the contact rate will be $U^{\prime} A N$, where $U^{\prime}$ is the random velocity, $A$ the feeding cross-section, and $\mathrm{N}$ the number density of food particles. The presence of turbulence greatly complicates this problem; nevertheless, the evaluation of components of random motion would seem to be a useful observation in studies of this type.

\section{Coherent structures and the distribution of plankton in a turbulent environment}

Small-scale studies of zooplankton distributions and behavior must be put in the context of the larger-scale motions in which they are embedded. Turbulence, and the dynamical conditions that lead to turbulence, are patchy and intermittent. Statistical measurements shed little light on, and can often obscure, the underlying structures of a turbulent flow. Thus, patches of turbulence need to be identified with the coherent features with which they are associated (shear flow instability, overturning, etc.). Acoustic images based on targetstrength distributions have proven very successful for defining these features. The targets are primarily zooplankton (or bubbles near the ocean surface), and the human eye is adept at recognizing the larger patterns and features of the flow. Several examples of such images have appeared in the literature (i.e., Farmer and Freeland, 1983). The resulting images not only show the physical structures, but can also lead to insights on the plankton behavior.

This list is by no means exhaustive and neglects some interesting, if relatively unproven, technologies such as acoustic holography. The potential for acoustic studies of plankton has further increased with the recent development of broad-band acoustic transducers and of increasingly sophisticated signal-processing concepts. However, it is essential to keep in mind that success in the application of these techniques depends critically on our understanding of the acoustic properties of the targets. The use of acoustic techniques for biological studies in the ocean is still in its infancy. The potential is great, but the need for careful experimental work, especially studies in laboratory conditions, is critical to its further development.

\section{Conclusion}

The study of fish recruitment has matured into the study of the interaction between density-dependent and physical factors occurring at all life-history stages. A coherent theory is required to "organize" the problem. Any theory will need to increase our understanding of the nonlinearities of the specific interactions between the physical setting and individual predator/prey interactions. Many difficult nonlinearities may be eliminated by considering the problem at its most fundamental level. This involves the specific role of each life-history stage and the individual feeding interaction between predator and prey. The individual feeding interactions can be thought of in terms of food signals which are amenable to further analysis, particularly with respect to turbulence at feeding scales. The theoretical analysis needs to be verified, and it seems at least from a preliminary perspective that "conventional" direct sampling devices will fall short of what is needed. Acoustic techniques appear to be promising, particularly inasmuch as the velocity of predator relative to the velocity of prey is important.

\section{References}

Andersen, R., and Zahuranec, B. J. (Eds.). 1977. Oceanic sound scattering predictions. Plenum, New York.

Clay, G.S., and Heist, B. G. 1984. Acoustic scattering by fish-acoustic models and a two-parameter fit. J. Acoust. Soc. Am., 75: 1077-1083.

Cox, D. R., and Isham, V. 1980. Point processes. Chapman and Hall, London. 188 pp.

Cushing, D. H. 1980. The decline of the herring stocks and the gadoid outburst. J. Cons. int. Explor. Mer, 39: 70-81.

Davies, J. T. 1972. Turbulence phenomena. Academic Press, New York.

Denbigh, P. N., and Weintraub, J. 1986. A statistical approach to fish stock assessment. Extended Abstract, 12th ICA Associated Symposium on Underwater Acoustics. Dept Engineering Physics, Technical University of Nova Scotia, pp. $41-42$.

Dickey, T.D., and Mellor, G. L. 1979. The Kolmogoroff $r^{2 / 3}$ law. Physics Fluids, 22: 1029-1032.

Dickey, T. D., and Mellor, G. L. 1982. Decaying turbulence in neutral and stratified fluids. J. Fluid Mech., 99: 13-31.

Farmer, D. M., and Freeland, H. J. 1983. The physical oceanography of fjords. Prog. Oceanogr., 12(2): 147-219.

Farmer, D. M., and Huston, R. D. 1988. Novel applications of acoustic backscatter to biological measurements. In Toward a theory on biological-physical interactions in the World Ocean, pp. 599-614. Ed. by B. J. Rothschild. Kluwer Academic Publishers, Dordrecht. 650 pp.

Granata, T. C., and Horne, A. J. 1985. Dispersion characteristics of two planktonic diatoms. J. Plankton Res., 7: 947-953.

Grant, J. L., Stewart, R. W., and Moillet, A. 1962. Turbulence spectra from a tidal channel. J. Fluid Mech., 12: 241-263.

Hinze, J. O. 1959. Turbulence. McGraw-Hill, New York. $586 \mathrm{pp}$.

Holliday, D. V., and Pieper, R.E. 1980. Volume scattering strengths and zooplankton distributions at acoustic frequencies between 0.5 and $3 \mathrm{MHz}$. J. Acoust. Soc. Am., 67: 135-146. 
Holliday, D. V., and Pieper, R. E. 1984. Acoustic volume scattering: $100 \mathrm{kHz}$ to $10 \mathrm{kHz}$. J. Acoust. Soc. Am., 75(2): $529 \mathrm{pp}$.

Holling. C. S. 1965. The functional response of invertebrate predators to prey density and its role in mimicry and population regulation. Mem. Entomol. Soc. Can., 45: 1-60.

Lueck, R. G., and Osborn, T. R. 1982. Dissipation measurements from the FRONTS- 80 expedition. Rep. 38, Dept of Oceanography, Univ. of British Columbia, Vancouver.

Lueck, R., and Reid, R. 1984. On the production and dissipation of mechanical energy in the ocean. J. geophys. Res., 89: 3439-3446.

Oakey, N. S., and Elliot, J. A. 1980. Dissipation in the mixed layer near Emerald Basin. In Marine turbulence. Ed. by J.C. J. Nihoul. Elsevier, New York.

Rothschild, B. J. 1986. Dynamics of marine fish populations. Harvard University Press, Cambridge, Massachusetts. $277 \mathrm{pp}$.

Rothschild, B. J. 1988. Biodynamics of the sea: The ecology of high dimensionality systems. In Toward a theory on biological-physical interactions in the World Ocean, pp. 527-548. Ed. by B. J. Rothschild. Kluwer Academic Publishers, Dordrecht. $650 \mathrm{pp}$.

Rothschild, B. J., and Fogarty, M. J. 1989. Spawning-stock biomass: A source of error in recruitment/stock relationships and management advice. J. Cons. int. Explor. Mer, 45: $131-135$.
Rothschild, B. J., and Osborn, T. R. 1986. Feasibility of relating recruitment to environmental variation. ICES CM 1986/L:25.

Rothschild, B. J., and Osborn, T. R. 1988. The effects of turbulence on planktonic contact rates. J. Plankton Res., 10(3): $465-474$.

Shepherd, J. G., and Cushing, D. H. 1980. A mechanism for density-dependent survival of larval fish as the basis of a stock-recruitment relationship. J. Cons. int. Explor. Mer, 39: $160-167$.

Stanton, T. K. and Clay, C. S. 1986. Sonar echo statistics as a remote-sensing tool: volume and seafloor. IEEE Journal of Oceanic Engineering, OE-11: 79-96.

Strickler, J. R. 1985. Feeding currents in calanoid copepods: Two new hypotheses. In Physiological adaptations of marine animals, pp. $459-485$. Ed. by M. S. Laverack. Symposia of the Society for Environmental Biology, 23. Pinder Group, Scarborough, North Yorkshire.

Wilhelmij, P., and Denbigh, P. 1973. A statistical approach to determining the number density of random scatterers from backscattered pulses. J. Acoust. Soc. Am., 76: 1810-1818.

Yamazaki, H., and Osborn, T.R. 1988. Review of oceanic turbulence: Implications for biodynamics. In Toward a theory on biological-physical interactions in the World Ocean, pp. 215-234. Ed. by B. J. Rothschild. Kluwer Academic Publishers, Dordrecht. 650 pp. 